\title{
Subcarrier Allocation Algorithm Based on Greedy-Like in Multi Carrier Code Division Multiple Access Systems
}

\author{
Muhammet Nuri Seyman ${ }^{1}$, Bircan Demiral ${ }^{2}$ \\ ${ }^{1}$ Kirikkale Vocational High School, Kirikkale University \\ 71450, Kirikkale, Turkey \\ ${ }^{2}$ Kazan Vocational High School, Baskent University \\ 06980, Ankara,Turkey \\ mnseyman@kku.edu.tr
}

\begin{abstract}
One of the most important issues to be taken into consideration in multi carrier communication systems with multi users is the assignment of the available resources to the users efficiently. Thus, how to determine the number of subcarriers to be allocated to the users has an important role with regards to data rate and error performance of the system when active users are increased in multi carrier system. In this study by using Greedy-Like algorithm, the subcarriers in MCCDMA systems are optimal allocated to the users and the efficiency of this algorithm compared with other algorithms in terms of bit error and bit rate performance.
\end{abstract}

Index Terms-MC-CDMA; subcarrier allocation; fairness; capacity maximization.

\section{INTRODUCTION}

The developments in the multimedia technologies during the recent years have brought along the need for high bit rate transmission. But the limitation of the available bandwidth has resulted in the development of multi carrier systems which use the existing bandwidth efficiently and by this means high bit rate data transmissions are provided. OFDM among the multi carrier systems is a technique that, along with allowing high bit rate data transmission by efficiently using the existing bandwidth, also increases the system's resistance against multi path fading. And CDMA is another multiplexing system which provides for the data transmission of multiple users at the same frequency and time domain using spreading codes. The multi carrier code division multiplexing technique (MC-CDMA) is established in order to use the advantages of OFDM and CDMA together. This technique is used as a multi user multiplexing method in wireless communication systems that allow high bit rate data transmission by utilizing subcarriers [1], [2]. But along with these mentioned advantages of the system, there is the problem to allocate subcarriers at the ratio required by the users in the system called subcarrier allocation [3]-[12].

When the subcarriers are not allocated to the users at the ratio required by them, there will be a decrease at the bit error rates on the receiver side, but at the same time the user data bit rates will also decrease. Subcarrier allocation

Manuscript received 29 September, 2016; accepted 2 April, 2017. algorithms are utilized in order to overcome this problem [3]-[12]. At the algorithms developed for subcarrier allocation are generally allocations processes like fairness, data speeds or channel capacity. In study [3], subcarriers that increase the bit rate of each user are allocated starting from the user with the minimal data speed in max-min algorithm suggested for multi user OFDM systems and by virtue of this the data speed per user is increased. And in study [4] the total channel capacity at MIMO-OFDMA systems is maximized and distributed to users in a fair manner proportional to the speeds of the subcarriers. Although many allocation algorithms are brought forward for the allocation of the resources, fairness criteria between users is not taken into consideration at these algorithms. Also in [5] resource allocation in MIMO-OFDMA systems is performed according to this fairness criterion. Although the performances shown by algorithms established according to the channel capacity and fairness criterion at OFDMA systems are high, it is seen that these type of allocation processes are not performed much at MC-CDMA systems.

In this study the subcarriers of MC-CDMA, are distributed among the users according to the fairness criterion and the increase of the system capacity is provided by utilizing the Greedy-Like algorithm. Thus the subcarriers are distributed to the users fairly, according to their need and both the bit error rates are decreased and the user bit rates are increased.

\section{SYSTEM MODEL OF MC-CDMA}

Primarily the data are converted from serial to parallel and spread with the pseudo code of the user in order to obtain the $Y(t)$ output of the MC-CDMA system with $M$ users and $N$ subcarriers for which the transmitter block is indicated in Fig. 1. Later the subcarriers are formed by converting the time domain from the frequency domain by the IFFT. After the cyclic prefix (CP) inserted to the symbols in order to decrease the inter-symbol interferences, the users' data are transmitted on the channel.

In this case, the $Y(t)$ output of an MC-CDMA system with $N$ subcarrier and $M$ users can be presented as indicated below. 

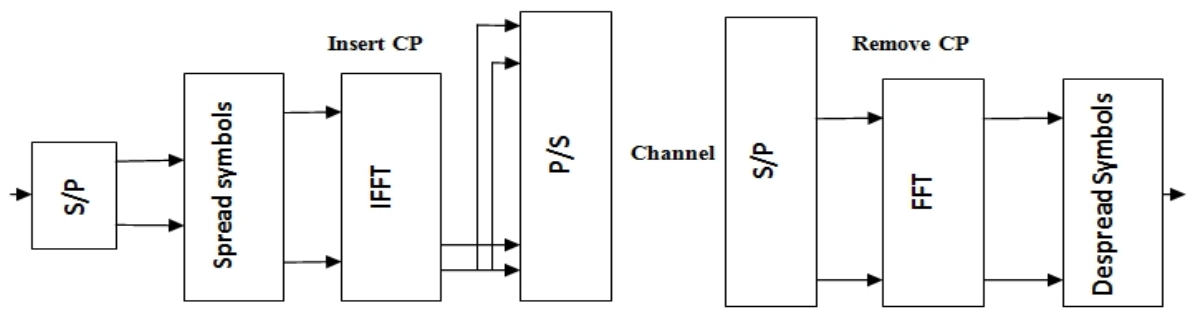

Fig. 1. MC-CDMA system.

$$
Y(t)=\sum_{m=1}^{M} A_{m} b_{m} \sum_{n=1}^{N} C_{m}(t) e^{j 2 \pi f_{n} t}+n_{w}(t),
$$

where $t[0, t]$.

In (1), $b_{m}$ is the data of user $m, A_{m}$ is the obtained signal amplitude of user $m, C_{m}(t)=c[m], c_{1}[m], c_{2}[m], \ldots, c_{L-1}[m]$ is $L$ bit length spreading code, $b_{m}$ is the data string of user $m$ and $n_{w}(t)$ is the white Gauss noise in the channel [1], [2].

When the total transmission power is accepted to be $P$, the transmission power for each of the $N$ number of subcarriers become $\frac{P}{N}$. At such a system, the data rate for user $m$ equation can be presented using (2)

$$
R_{m}=\frac{B}{N} \sum_{n=1}^{N} v_{m, n} \log _{2}\left(1+\gamma_{m, n}\right)
$$

where $\frac{B}{N}$ is the MC-CDMA symbol time, $\gamma_{m, n}$ is the parameter indicating whether, $n$ subcarrier will be allocated to user $k$, and if it is allocated, it gets the value 1 , and in case of an non-allocation is its value 0 . And $\gamma_{m, n}$ is the $n$ subcarrier's signal/noise ratio for user $m$

$$
\gamma_{m, n}=\frac{P\left|h_{m, n}\right|^{2}}{B N_{0}},
$$

where $P$ is the transmission power, $h_{m, n}$ is $m_{t h}$ channel gain of user $m$ at the $n$ subcarrier, $B$ is the bandwidth and $N_{0}$ is the power spectral density.

We use the (4) in order to allocate the subcarrier optimally to the users

$$
\max =\sum_{m=1}^{M} \sum_{n=1}^{N} v_{m, n} \log _{2}\left(1+\gamma_{m, n}\right),
$$

where $v_{m, n}$ the parameter whether subcarrier $n$ can be allocated to user $m$ or not. This expression is indicated as in (5):

$$
\begin{gathered}
v_{m, n} \in\{0,1\}, \forall m, n, \\
\sum_{n=1}^{N} v_{m, n}=1, \forall n,
\end{gathered}
$$

Equations (5) and (6) are the necessary conditions indicating which subcarrier is allocated to which user during subcarrier allocation [3].

\section{GREEDY-LIKE ALGORITHM FOR MC-CDMA}

In this algorithm the allocation process is performed in
2 steps. Generally, the number of the subcarriers for each user is determined and the subcarriers are allocated to the users at the first and second steps respectively.

Step 1. Determination of the number of subcarriers to be allocated per user

At the beginning, number of subcarriers $N_{m}$ is determined for each user. The channel gain of the algorithm established depending on the gain is indicated in (7)

$$
\overline{H_{m}}=\frac{1}{N} \text {. }
$$

The data rate is determined by (8) when total $P$ power is allocated to each user in order to decrease any allocation complexity

$$
\overline{R_{m}}=\frac{N_{m}}{N} \log _{2}\left(1+\frac{\overline{H_{m} P_{m}}}{N_{0}}\right)
$$

The algorithm of this step is as indicated below.

a) BEGIN

1) For the start, designate the number of the subcarriers to be assigned to the users.

$$
N_{m}=\gamma_{m}, m=1,2, \ldots, M \quad N_{\text {assign }}=\sum_{m=1}^{M} \gamma_{m}
$$

b) Calculate the channel gain for each user by (7).

1) Perform equal power allocation $\overline{P_{m}}$ between subcarriers by $N_{\text {assign }}<N_{m}$

2) Update $\overline{R_{m}}$ with (8).

c) While $N_{\text {assign }}<N_{m}$

1) Find $\hat{m}=\arg \min _{m} \frac{\overline{R_{m}}}{\gamma_{m}} \quad m=1,2, \ldots, M$

$$
N_{\widehat{m}}=N_{\widehat{m}}, N_{\text {assign }}=N_{\text {assign }}+1
$$

2) Allocate the equal power between the subcarriers $\overline{P_{m}}$ by $N_{\text {assign }}$

3) Update the $\overline{R_{m}}$

Step 2. Subcarrier Allocation

At this step by maximizing the system capacity, assignment process is performed while keeping the sharing rate between the users fixed. According to this the algorithm is indicated as below.

a) BEGIN

1) Sort the users according to their average gains.

$$
\overline{H_{1}} \leq \overline{H_{2}} \leq \ldots \leq \overline{H_{g}} \leq \ldots \leq \overline{H_{m}}
$$

2) Separate the users as two groups according to 
their channel gains.

Group with bad channel gains: user $_{b}=\{1,2, \ldots, g\}$

Group with good channel gains:

$$
\text { user }_{\mathrm{j}}=\{g+1, g+2, \ldots M\}
$$

b) for $m=0$ to $G$

1) Find subcarrier $n$ such to provide equation

$$
\prod_{i=1}^{G_{m, n}} \lambda_{m, n}^{i} \geq \prod_{i=1}^{G_{m, j}} \lambda_{m, j}^{i} \quad j \in A
$$

2) Subtract subcarrier $m$ from cluster and decrease the number of subcarriers to be assigned by one. Later, update equation $R_{m}$

$$
A=A-1, N_{m}=N_{m}-1
$$

c) While $|A|>N-\sum_{i=1}^{l} N_{i}$

1) $\operatorname{user}_{\mathrm{b}}=\{1,2, \ldots, g\}$

Find the user $m$ providing $\frac{R_{m}}{\gamma_{m}} \leq \frac{R_{i}}{\gamma_{i}}, \quad 1 \leq i \leq g$

2) Determine from the equation the subcarrier $n$ for the found user $m$

$$
\prod_{i=1}^{G_{m n}} \lambda_{m n}^{i} \geq \prod_{i=1}^{G_{m j}} \lambda_{m j}^{i} \quad j \in A
$$

3) If $N_{m}>0$

Subtract that subcarrier from cluster $A$, decrease the total number of the subcarriers by one and update $R_{m}$

$$
A=A-1, \quad N_{m}=N_{m}-1
$$

Else;

user $_{\mathrm{b}}=$ user $_{\mathrm{b}}-\mathrm{m}$

The allocation process will be completed when step $b$ and step c are performed for user $s$ too.

\section{Simulation Results}

The simulations are performed using simulation parameters in Table I with Matlab 2012.

TABLE I. SIMULATION PARAMETERS

\begin{tabular}{|c|c|}
\hline Parameter & Value \\
\hline Total Power & $1 \mathrm{~W}$ \\
\hline Bandwidth & $1 \mathrm{MHz}$ \\
\hline Number of Subcarrier & $64 / 128 / 256 / 512$ \\
\hline Coding Type & Walsh Hadamard \\
\hline Modulation Type & BPSK \\
\hline Channel Type & 4 Tab Multipath Rayleigh Fading \\
\hline
\end{tabular}

Figure 2 indicates the performance assessment of the Greedy-Like algorithm proposed for subcarrier allocation for a MC-CDMA system with 5 users and different subcarrier numbers.

As to be seen from the Fig. 2; that the BER values decreases and the system performance increases in case of number of the subcarriers in the system is increased. The reason is, the fast fading effect generated by the channel decreases and the channel returns to flat fading condition when the number of the subcarriers allocated to the users is high.

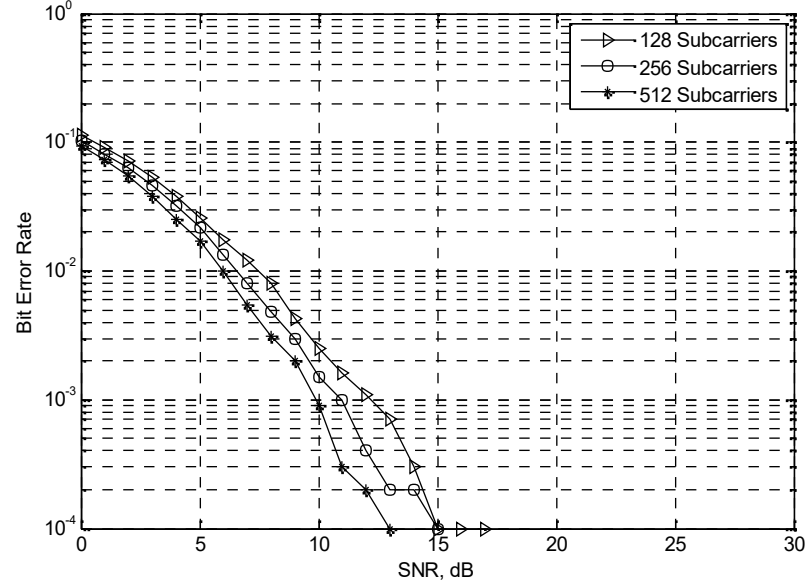

Fig. 2. BER of Greedy-Like algorithm versus various subcarriers for 5 users.

And this situation indicates that the importance of the appropriate the subcarrier allocation performance on the system's performance for cases where the number of the users increases. Examining Fig. 2, it is to be seen that the performance of the proposed algorithm in a system with 128 subcarriers, where the number of the subcarriers is low, is lower compared with systems with a higher number of subcarriers. For example, a system with 128 subcarriers will have an SNR loss of nearly $1 \mathrm{~dB}$ compared with a system with 256 subcarriers in order to obtain a BER value of $10^{-2}$ This loss further increases at lower BER values. While the SNR difference of a system with 256 subcarriers at $10^{-2}$ BER value compared with a system with 512 subcarriers is nearly less than $1 \mathrm{~dB}$, the difference increased up to $1.5 \mathrm{~dB}$ at $10^{-3} \mathrm{BER}$ value.

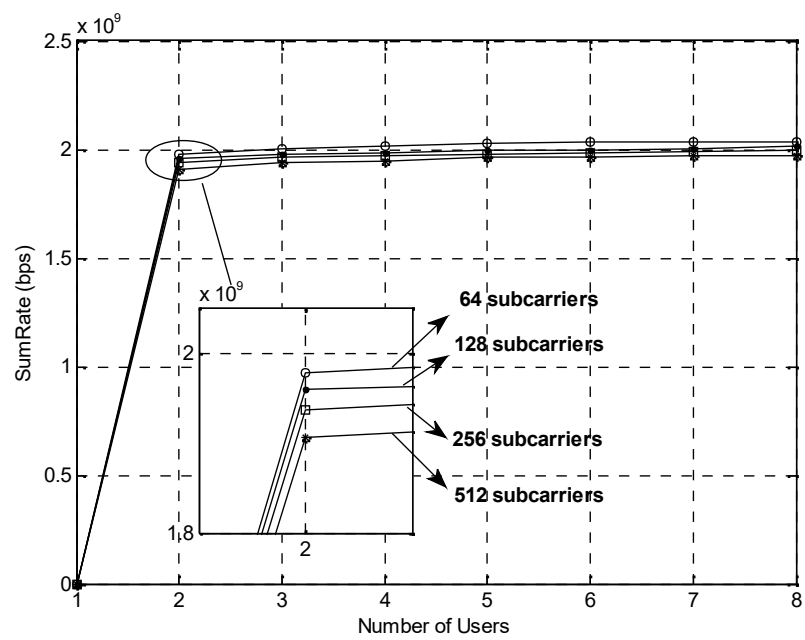

Fig. 3. Sum rate of various subcarriers.

In Fig. 3, the total bit rate performance of the GreedyLike algorithm in a system with different numbers of users and subcarriers. As to be seen in Fig. 3, the total bit rate increases depending on the increase of the number of the subcarriers. And the system's bit rate is higher compared with systems with 256 and lesser subcarriers when the number of the subcarriers is 512. Thanks to the Greedy-Like algorithm, there wasn't decrease at bit rates parallel to the increase of the number of the users.

Figure 4 includes the BER performance assessment for the $1_{\text {st }}$ user of different subcarrier allocation algorithms providing the allocation of 512 subcarriers to 3 users in MC- 
CDMA systems.

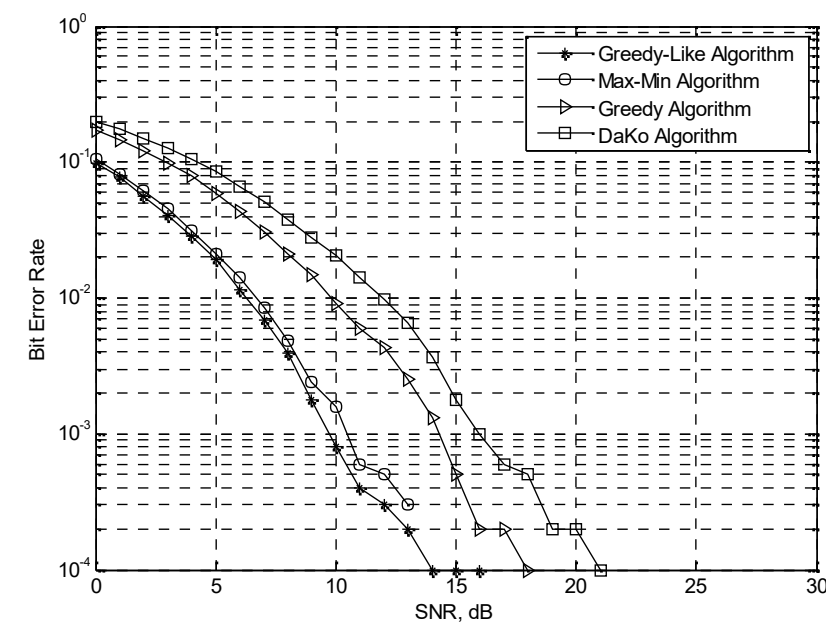

Fig. 4. BER of algorithms for 3 users and 512 subcarriers.

According to Fig. 4; it is to be seen that the Greedy-Like algorithm performs best and the Da-Ko algorithm performs worse. Greedy-Like algorithm provides an SNR gain of nearly $3 \mathrm{~dB}$ at the BER value $10^{-2}$ compared with the Greedy algorithm, this gain has a value of nearly $5 \mathrm{~dB}$ compared with the Da-Ko algorithm. And the SNR difference between the Greedy algorithm and the Da-Ko algorithm is $2.5 \mathrm{~dB}$. Although the Max-Min algorithm is an algorithm showing a better performance than both the Greedy algorithm and the Da-Ko algorithm, it has a worse performance than the Greedy-Like algorithm. And the performance of the Greedy-Like algorithm further increases where the SNR value increases.

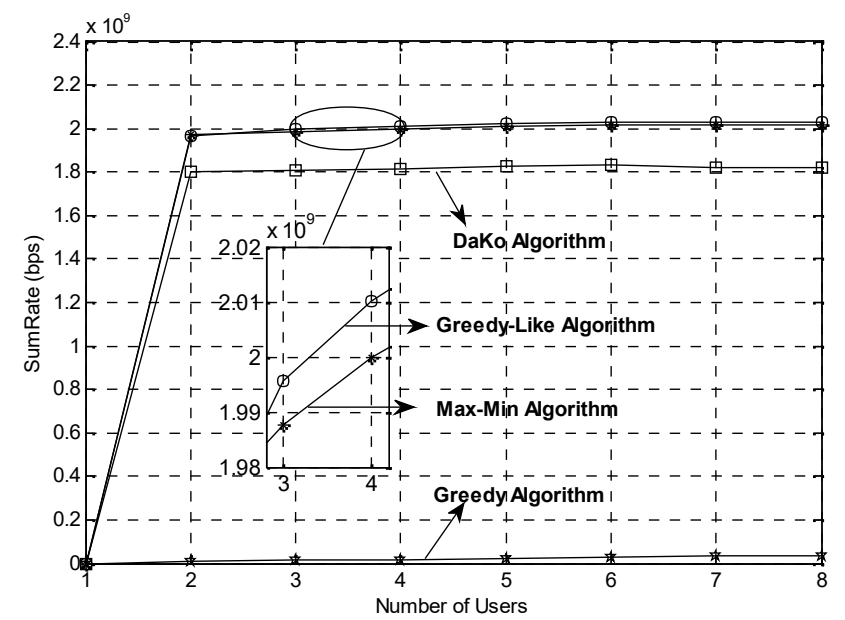

Fig. 5. Sum rate of algorithms.

Figure 5 indicates the total bit rate performances of the algorithms in a system with 512 subcarriers against the increase of the number of the users. The proposed GreedyLike algorithm shows a better performance than the other algorithms in terms of total bit rate. For example, while Greedy-Like Greedy Like has a rate of $2.1 \times 10^{9} \mathrm{bps}$ in a system with 4 users, the bit rate of the Max-Min algorithm with the closest bit rate to this algorithm is $2.0 \times 10^{9} \mathrm{bps}$. The bit rate of the Da-Ko algorithm under the same conditions is $1.82 \times 10^{9} \mathrm{bps}$. And the worst bit rate value has the Greedy algorithm.

\section{CONCLUSIONS}

Subcarrier allocation is an important scheme for multi user multi carrier systems like MC-CDMA in terms of bit error rate and speeds of the systems. Therefore, Greedy-Like algorithm is proposed for subcarrier allocation in MCCDMA systems and the performance of this algorithm is compared with other allocation algorithms. The most important feature of this algorithm different from other algorithms, it performs allocation process by utilizing not only capacity but also fairness criteria. Thereby better BER and total bit rate values than other algorithms are obtained. Simulations at systems with high number of subcarriers are performed in order to show the efficiency of this proposed algorithm. From the obtained results of BER graphic, it is seen that Greedy-Like algorithm has higher SNR gain than other algorithms. Also sum rate of the system which uses this proposal is better than others.

\section{REFERENCES}

[1] S. Hara, R. Prasad, "Overview of multicarrier CDMA", IEEE communication magazine, vol. 35, no. 12, pp. 126-133, 1997. [Online]. Available: http://dx.doi.org/10.1109/35.642841

[2] R. Craciunescu, C. Voicu, A. Vulpe, S. Halunga, "Performance analysis of MC-CDMA system when image transmission is involved", in 10th Int. Conf. Communications (COMM), 2014, pp. 14. [Online]. Available: http://dx.doi.org/10.1109/ICComm. 2014.6866742

[3] H. Rasouli, A. Anpalagan, "An asymptotically fair subcarrier allocation algorithm in OFDM systems", in Proc. Conf. 69th IEEE Vehicular Technology Conf., Barcelona, 2009, pp. 1-5. [Online]. Available: http://dx.doi.org/10.1109/VETECS.2009.5073378

[4] Z. Shen, J. G. Andrews, B. L. Evans, "Adaptive resource allocation in multiuser OFDM systems with proportional rate constraints", IEEE Trans. Wireless Communications, vol. 4, no. 6, pp. 2726-2736, 2005. [Online]. Available: http://dx.doi.org/10.1109/TWC.2005.858010

[5] B. Da, C. C. Ko, Y. Liang, "An enhanced capacity and fairness scheme for MIMO-OFDMA downlink resource allocation", in Proc. Int. Symp. on Communications and Information Technologies, Sydney, NSW, Australia, 2007, pp. 495-499. [Online]. Available: http://dx.doi.org/10.1109/ISCIT.2007.4392069

[6] J. Xu, J. Kim, W. Paik, J. S. Seo, "Adaptive resource allocation algorithm with fairness for MIMO-OFDMA system", in Proc. Conf. 63rd IEEE Vehicular Technology Conf., Melbourne, 2006, pp. 15851589. [Online]. Available: http://dx.doi.org/10.1109/VETECS. 2006.1683113

[7] A. Yaqot, P. Hoeher, "Efficient resource allocation in cognitive networks", IEEE Trans. Vehicular Technology, vol. 66, no. 7, pp. 6349-6361, 2016. [Online]. Available: http://dx.doi.org/10.1109/ TVT.2016.2642785

[8] N. Sharma, A. S. Madhukumar, "Genetic algorithm aided proportional fair resource allocation in multicast OFDM systems", IEEE Trans. Broadcasting, vol. 61, no. 1, pp. 16-29, 2015. [Online]. Available: http://dx.doi.org/10.1109/TBC.2015.2389692

[9] M. Al-Imari, Pei Xiao, M. A. Imran, R. Tafazolli, "Low complexity subcarrier and power allocation algorithm for uplink OFDMA systems", EURASIP Journal on Wireless Communication and Networking, vol. 98, pp. 1-6, 2013. [Online]. Available: https://doi.org/10.1186/1687-1499-2013-98

[10] A. C. Cirik, K. Rikkinen, Y. Rong, T. Ratnarajah, "A subcarrier and power allocation algorithm for OFDMA full-duplex systems", European Conf. Networks and Communications (EuCNC 2015), 2015, pp. 11-15. [Online]. Available: https://doi.org/10.1109/ EuCNC.2015.7194031

[11] M. N. Seyman, B. Sacakli, "A novel subcarrier allocation algorithm for MC-CDMA systems", Journal of Electrical Engineering, vol. 67, no. 5, pp. 371-376, 2016. [Online]. Available: https://doi.org/ $10.1515 /$ jee-2016-0053

[12] S. Yin, Z. Qu, "Resource allocation in multiuser OFDM systems with wireless information and power transfer sign in or purchase", IEEE Communications Letters, vol. 20, no. 3, pp. 594-597, 2016. [Online]. Available: https://doi.org/10.1109/LCOMM.2016.2516999 